\title{
Are magicians specialists at identifying deceptive motion? The role of expertise in being fooled by sleight of hand.
}

Elias Garcia-Pelegrin ( $\nabla$ eg573@cam.ac.uk)

University of Cambridge

Clive Wilkins

University of Cambridge

Nicola Clayton

University of Cambridge

\section{Research Article}

Keywords: magic, perception, sleight of hand, expertise, magician

Posted Date: September 21st, 2021

DOI: https://doi.org/10.21203/rs.3.rs-818944/v3

License: (c) (i) This work is licensed under a Creative Commons Attribution 4.0 International License.

Read Full License 


\section{Abstract}

The use of magic effects to investigate the blind spots in the attention and perception and roadblocks in the cognition of the spectator has yielded thought-provoking results elucidating how these techniques operate. However, little is known about the interplay between experience practising magic and being deceived by magic effects. In this study, we performed two common sleight of hand effects and their real transfer counterparts to non-magicians, and to magicians with a diverse range of experience practising magic. Although, as a group, magicians identified the sleights of hand as deceptive actions significantly more than non-magicians; this ability was only evidenced in magicians with more than 5 years in the craft. However, unlike the rest of the participants, experienced magicians had difficulty correctly pinpointing the location of the coin in one of the real transfers presented. We hypothesise that this might be due to the inherent ambiguity of this transfer, in which, contrary to the other real transfer performed, no clear perceptive clue is given about the location of the coin. We suggest that extensive time practising magic might have primed experienced magicians to anticipate foul play when observing ambiguous movements, even when the actions observed are genuine.

\section{Main Text}

Magicians use intricate techniques of deception that capitalise on the spectator's blind spots in attention and perception and roadblocks in cognition (Clayton \& Wilkins, 2019; Garcia-Pelegrin et al., 2020; Kuhn et al., 2008). The interest in how magic effects exploit such psychological constraints has been recently growing within the psychological community, and more frequently new evidence regarding the qualities that make the observer liable to these techniques is being unearthed. For example, when observing sleight of hand, curved motions appear to have a larger effect in misdirecting the observer than utilising rectilinear motions (Otero-Millan et al., 2011). While the use of social misdirection techniques such as joint attention might strengthen a magic effect (Kuhn \& Land, 2006), these techniques do not seem to be imperative for their success (Cui et al., 2011; Thomas \& Didierjean, 2016). However, although the investigation of magic effects has produced thought-provoking results on how the techniques operate on the spectator in both humans and non-human animals (see Garcia-Pelegrin et al., 2021), the investigation of how practising magic affects magicians' perceptual and cognitive abilities merits further exploration.

To become proficient at reliably fooling their audience, most magicians spend years training in the craft through meticulous deliberate practice (Corrieri, 2016; Rissanen et al., 2014). Attaining a high level as a magician is not an easy task, for example out of an average of 1500 professional magicians at The Magic Circle@ only $20 \%$ of them have reached the highest rank (Gold star). Magicians' extensive training in sleight of hand grants them very specific motor-performance skills (Green et al., 2013; Harte \& Spencer, 2014), which allows them to seemly mislead their spectators into experiencing the impossible. Indeed, it is these abilities that make them experts at manipulating their audience's perception and attention (Barnhart, 2010; Kuhn et al., 2014). Consequently, one might justifiably consider the study of magicians to inherently be the study of expertise. 
Experts (i.e., those who, through practice, have perfected a particular domain) have been the topic of interest of scientists for centuries (e.g. Galton, 1869), and some have aimed to disentangle whether expertise in a particular domain is a transferable skill and therefore granted the experts with superior ability in a different array of perceptual tasks (Ericsson \& Smith, 1991; Ericsson, 1996; Glaser \& Chi, 1988). Research investigating the abilities gained through expertise across different domains has yielded significant results in many areas of expertise such as chess (Charness et al., 2005; Gobet \& Charness, 2018), music (Gaser \& Schlaug, 2003; Morreale et al., 2013; Wöllner \& Cañal-Bruland, 2010), sport (Camponogara et al., 2017; Mann et al., 2007; Moore et al., 2019; Williams et al., 2002), and even medicine (Ericsson, 2004). It is currently well established that while experts elicit superior performance over novices in domain-related tasks, they do not tend to significantly differ outside of the construct of the domain in which they are experts in (Ericsson, 2004; Kirkman, 2013; Ward et al., 2007). For example, when testing experienced handball goalkeepers and novices in a reaction time task that was directly related to handball goalkeeping (moving the right or left hand towards a target in a handball goal), the experienced goalkeepers demonstrated a superior perceptual and reactionary ability in comparison to the novices. However, when tested in a similar yet not specific to goalkeeping task, the expert group did not show a significant effect of expertise in their reaction times when compared to novices (Helm et al., 2016). The occurrence that experts gain superior perceptual abilities concerning the domain they regularly practice is understandable. The close link between action and perception is hardly up for debate (Calvo-Merino et al., 2005; Gallese \& Goldman, 1998; Rizzolatti et al., 2001), and this relationship is further pronounced in the role of expertise, as expert behaviours are mostly directed towards specific targets. As such, perceptual mechanisms in experts tend to adapt to the niches of the action patterns that are performed regularly (Ericsson, 2012). The evidence that learning in some domains can affect the visual recognition of specialized patterns is evidenced in dentistry (Hegarty et al., 2009), chemistry (Stull et al., 2012), meteorology (Hegarty et al., 2010), and geology (Shipley et al., 2009) to name but a few examples.

Therefore, one might pose the question as to whether magicians, whose expertise involves being highly adept at object manipulation and action mimicry, display domain expertise in such areas when compared to non-magicians. Evidence from expertise studies in magicians reveals that magicians appear to excel in comparison to non-magicians in several faculties. For example, when observing video recordings of people performing reach to grasp actions, sleight of hand practitioners evidenced an enhanced ability to discriminate real from pantomimed actions when compared to a sample of non-magicians (Quarona et al., 2020). Moreover, in comparison to non-magicians, magic practitioners appear to excel at producing pantomimed grasping actions of objects (but only when the said object is being observed while producing the pantomime) (Cavina-Pratesi et al., 2011) and appear to have a more sophisticated mental hand representation (Cocchini et al., 2018). Consequently, the laborious training in sleight of hand that magicians undergo seems to grant their perpetrators an advantage when observing and performing manual manoeuvres. However, little is known about the interplay between the blind spots in perception exploited by sleight of hand effects and their relationship with magicians' expertise. Sleight of hand techniques are particularly powerful as they capitalise on the spectators' expectations of typical object exchanges between hands. Manipulations of objects between hands are typically learned early in 
development, performed regularly, and seldom pondered upon once mastered (Vauclair \& Bard, 1983). Similarly, when observing others moving objects between hands, we use our previous experience of object manipulation and observation of others manipulating to assume the most likely outcome (i.e., that the object has been transferred successfully), thus completing the movement even when we cannot explicitly see it (i.e., amodal completion (Kanizsa \& Gerbino, 1982; Michotte et al., 1991)), and, when observing sleight of hand, missing the potential cues that might reveal foul play (Ekroll et al., 2013; Ekroll \& Wagemans, 2016).

In this study, we investigated whether magicians were more proficient at identifying sleight of hand movements from real transfers of objects than non-magicians. We performed two common sleight of hand techniques and their real transfer counterparts to a sample of 165 non-magicians, and a sample of 93 magicians with different expertise levels. The sleight of hand techniques used (palming and French drop) are typically employed to mislead the spectators into believing one object has successfully been transferred from one hand to the other, while in truth the object remains concealed in the initial hand. These are common methodologies used in most close-up magic routines and typically learnt early on when studying sleight of hand. While similar in its importance for a good grasp of magic performance, both palming and French drop techniques differ greatly in the visual cues given to the observer. Palming involves the retention of an object between the palmar muscles of the hand whilst simultaneously mimicking the transfer of the object from one hand to the other. In contrast, the version of the French drop used in this study involves mimicking the grab of the object with one hand, whilst simultaneously dropping the object so that it falls out of the hand that was holding it (Fig. 1). Given the plethora of evidence suggesting that experts in one domain tend to excel at perceptive tasks which are related to such domain, we hypothesise that magicians will outperform non-magicians at differentiating real from sleight of hand transfer.

\section{Methods}

The choices of the 165 non-magicians were obtained prior to this study for a different experiment that involved the participants observing three magic effects (Palming, French drop, and Fast Pass) and a subsequent comparison with the choices of a sample of Eurasian jays (Garrulus glandarius) (see supplementary information in Garcia-Pelegrin et al., 2021). The sample of magicians $(n=93)$ was recruited from The Magic Circle $\odot$, the Leicester Magic Circle, and the Cambridge University Magic Society social media platforms and all of these participants were provided with access to the survey via a link. Both magicians' and non-magicians' surveys contained the exact same videos of the French drop and palming magic effects and real transfer counterparts. Participants in the non-magician survey observed an extra magic effect and an extra control condition per effect, which were performed specifically for the comparison with non-human animals, thus being irrelevant to the purpose of this study and omitted for the magician survey. Both surveys were designed using Qualtrics, the magician survey consisted of 4 videos for each of the two experiments (i.e., 2 videos per magic effect, 2 videos per real transfer counterpart, each one being performed right to left, and vice versa), the non-magician survey consisted of the same videos and arrangement plus the extra unrelated magic effect and control conditions. The 
videos of the effects consisted of purposely pre-recorded videos of the experimenter's hands performing the effects, the experimenter had an $\mathbf{O}$ (left hand), or $\mathbf{X}$ (right hand) painted on the back of each corresponding hand for better identification by the participant. Participants were asked to attentively observe each video of the effect and then identify which hand contained the coin by choosing $\mathbf{O}$ or $\mathbf{X}$ appropriately. The order of the videos was randomised for each candidate. All participants were provided with the relevant information of the study and gave their informed consent prior to their participation,

\section{Ethical approval}

As the experiment did not involve the subjects disclosing any information about their individual identity, the participants were not from any vulnerable group, and the conditions of the experiment did not pose any risk to the participant, the experiment did not require ethical approval by the University of Cambridge. All procedures were performed in accordance with relevant guidelines.

\section{Conditions}

\section{Experiment 1}

\section{Palm transfer}

In this condition, the experimenter utilised a palming technique to simulate a coin being transferred from one hand to the other. The technique involves the concealment of the coin in the middle of the palm, held together by loosely clenching both the right and left sides of the palm (i.e., the thenar and hypothenar muscles). When properly performed, the magician takes an object with one hand, secures it in their palm, and then fake transfers it to the other hand whilst simultaneously pretending to catch the object, even though in reality, the object remains in the original hand. To the naïve spectator, however, it will appear to be a seamless transfer of objects from one hand to the other.

\section{Real palm transfer counterpart}

In the real transfer condition for this experiment, the coin was genuinely transferred from one hand to the other. The experimenter recreated the same movements that were used in a Palm transfer but allowed the coin to drop to the opposing hand, instead of retaining it in the palm. Note that in contrast with the magic effect version, in this transfer the coin could be seen being dropped from one hand to the other.

\section{Experiment 2}

\section{French drop}

The French drop condition also relied on another fake transfer technique used by magicians. This method of sleight of hand consists of holding a coin in one hand and then mimicking a grabbing motion of the coin that is held between the thumb and both the index and middle finger of the opposite hand, creating the illusion that the coin has been transferred from one hand to the other. This sleight utilises the hand mimicking the grab of the coin to occlude how the coin is dropped inside the palmar region of the original 
hand holding it, while simultaneously mimicking how the grasping hand enacts a precision grip on the same coin.

\section{French drop's real transfer counterpart}

The French drop's real transfer condition simulated the same movements as the French drop condition without allowing the coin to drop to the palm of the hand holding it. Thus, the coin was grabbed by the hand performing the precision grip and genuinely transferred from one hand to the other. In order to keep both movements as similar as possible, the coin was still occluded by the grasping hand when grabbed. Note that in neither of the conditions of this experiment did the coin appear in view after being occluded by the grasping hand.

\section{Analysis}

The data was analysed using JASP (v.0.10.3, http://jasp-stats.org) and RStudio for Mac (version 1.2.1335). To determine the subjects' choices per condition and their relationship to both treatment and experience as explanatory variables we used binomial tests (against value: 0.5 ), and performed generalized linear mixed models (GLMM) with subject specified as a random term in the model to control for repeated measures (Schall, 1991). Significant differences between treatments were further explored using post-hoc pairwise comparisons and were adjusted using the Holm-Bonferroni method to maintain the overall alpha level at the nominated value or 0.05 for multiple pairwise comparisons.

\section{Results}

\section{Experiment 1: Palm transfer}

\section{Non-magicians versus Magicians.}

As shown in Figure 2, in Experiment 1 both non-magicians and magicians significantly chose the correct hand when observing the real transfer counterpart (binomial test: $p<0.01$ for both). However, only magicians significantly chose the correct hand when observing a palm transfer (binomial test. $p<0.01$ ), while non-magicians significantly choose incorrectly (binomial test. $p<0.01$ ) i.e., non-magicians were significantly misled by the motions of the magic effect, whereas the magicians were not.

A GLMM revealed that there was a significant effect on the participants' choices for condition $\left(\chi^{2}=\right.$ $135.695 ; \mathrm{df}=1 ; p<0.001)$, significant effect for skill (i.e., non-magician or magician) $\left(\chi^{2}=26.134 ; \mathrm{df}=1\right.$; $p<0.001)$, and significant interaction between condition and skill $\left(\chi^{2}=31.701 ; \mathrm{df}=1 ; p<0.001\right)$. Posthoc pairwise comparisons with the Holm-Bonferroni adjustment showed that both non magicians and magicians' choices in the real transfer significantly differed from their respective choices in the palm transfer ( $p<0.001$ for both). Non-magicians and magicians' choices did not significantly differ in the real transfer $(p=0.07)$, but they did so in the palm transfer $(p<0.001)$. 


\section{Effect of experience practising magic.}

In order to investigate the effects of experience on the participants' choices, the magician's group was split into three levels of experience ( 1 to 4 years $(n=56) ; 5$ to 10 years $(n=70)$; and > 10 years $(n=60)$ ). Binomial tests showed that all participants were more likely to choose the correct hand when observing a real transfer independently of their level of experience performing magic (binomial test. $p<0.001$ ). When observing a palm transfer, however, non-magicians, and magicians with 1 to 4 years of experience significantly chose incorrectly (binomial test: $p<0.001$, and $p=0.044$ respectively). By contrast, magicians with 5 to 10 years of experience and more than 10 years of experience significantly chose correctly (binomial test: $p<0.001)$ (Figure. 3).

A GLMM showed that there was a significant effect of condition $\left(\chi^{2}=118.347 ; \mathrm{df}=1 ; \mathrm{p}<0.001\right)$, a significant effect of experience practicing magic $\left(\chi^{2}=26.210 ; \mathrm{df}=3 ; p<0.001\right)$, and a significant interaction between condition and experience $\left(\chi^{2}=46.508\right.$; $\left.\mathrm{df}=3 ; p<0.001\right)$. To investigate this further a series of post-hoc pairwise comparisons with the Holm-Bonferroni adjustment were performed. The choices of all magicians significantly differed between their choices in the real transfer and their respective choices in the palm transfer $(p<0.001$ for all). There was no significant difference in the palm transfer condition between non-magicians and magicians with 1 to 4 years of experience $(p=1)$, and no significant difference between magicians with 5 to 10 years of experience and magicians with more than 10 years $(p=1)$. When observing a palm transfer, both the choices of magicians with 5 to 10 years of experience and magicians with more than 10 years significantly differed from non-magicians' choices ( $p$ $<0.001$ for both), and the choices of magicians with 1 to 4 years of experience ( $p<0.001$ for both). There was no significant difference in the real transfer condition between non magicians and magicians irrespective of their level of experience performing magic.

\section{Experiment 2: French drop}

\section{Non-magicians vs Magicians.}

Figure 4 shows the choices for both non-magicians and magicians in Experiment 2, in which both nonmagicians and magicians significantly choose correctly when observing a real transfer (binomial test. $p<$ 0.001 for both). However, non-magicians significantly chose incorrectly when observing a French drop (binomial test. $p<0.001$ ), while magicians significantly chose correctly (binomial test. $p=0.01$ ).

A GLMM showed a significant effect in the participants' choices for condition $\left(\chi^{2}=108.9570 ; \mathrm{df}=1 ; p<\right.$ $0.001)$, but not for skill $\left(\chi^{2}=1.2709 ; \mathrm{df}=1 ; p=0.25\right)$, and a significant interaction between condition and level $\left(\chi^{2}=72.2841 ; \mathrm{df}=1 ; p<0.001\right)$. Non magician's choices significantly differed between the real transfer and palm transfer $(p<0.001)$. There was no significant difference between the magicians' choices in the real transfer and French drop $(p<0.33)$. There was a significant difference between nonmagicians' and magicians' choices in the real transfer $(p<0.001)$ and their choices in the French drop $(p$ $<0.001)$. 


\section{Effect of experience practising magic.}

Similarly to Experiment 1, to investigate the effect of experience practising magic in the participants' choices, the magician's group was split into their levels of experience. As shown in Figure 5, when observing the real transfer, non-magicians, and magicians with 1 to 4 years of experience were more likely to choose the correct hand (binomial test: $p<0.001$ and $p=0.005$ respectively). There was no significant difference in the choices of magicians with 5 to 10 years of experience (binomial test: $p=0.72$ ), and more than 10 years of experience (binomial test: $p=0.89$ ). Magicians with 1 to 4 years of experience were more likely to choose incorrectly when observing a French drop (binomial test: $p<0.001$ for both). Conversely, magicians with more experience were more likely to choose correctly (5 to 10 years of experience and magicians with more than 10 years of experience (binomial test. $p<0.001)$.

The GLMM revealed that there was a significant effect in the participants' choices for condition $\left(\chi^{2}=\right.$ 95.6946; $\mathrm{df}=1 ; p<0.001)$, and a significant interaction between condition and experience $\left(\chi^{2}=135.0624\right.$; $\mathrm{df}=3 ; p<0.001)$. Binomial tests were conducted to investigate the number of correct vs incorrect choices per group, and a series of post-hoc pairwise comparisons with the Holm-Bonferroni adjustment were performed. Magicians' with 1 to 4 years of experience choices significantly differed between the real transfer and French drop $(p<0.001)$. Magicians' with 5 to 10 years of experience and magicians' with more than 10 years of experience choices did not significantly differ between the real transfer and French drop ( $p=0.059$ and $p=0.12$ respectively $)$.

There was no significant difference in the real transfer condition between non magicians and magicians with 1 to 4 years of experience $(p=0.8)$, and there was no significant difference in the real transfer condition between magicians with 5 to 10 years of experience and magicians with more than 10 years of experience $(p=1)$. However, the choices of both magicians with 5 to 10 years of experience and magicians with more than 10 years significantly differed from non-magicians' choices $(p<0.001$ for both), and magicians with 1 to 4 years of experience ( $p<0.001$ for both). There was no significant difference in the French drop condition between non-magicians and magicians with 1 to 4 years of experience $(p=1)$, and no significant difference between magicians with 5 to 10 years of experience and magicians with more than 10 years of experience $(p=1)$. Both the choices of magicians with 5 to 10 years of experience and magicians with more than 10 years significantly differed from non-magicians' choices ( $p<0.001$ for both), and magicians with 1 to 4 years of experience ( $p<0.001$ for both).

\section{Discussion}

In this study, magicians were better than non-magicians at differentiating real from fake transfers using both the palm transfer (Experiment 1) and the French drop (Experiment 2). Furthermore, there was a clear relationship between the years of experience practising magic and the ability to detect sleight of hand effects. As expected, spectators of magic effects with no prior engagement in the craft were not able to differentiate a fake transfer from a real one in either experiment. 
There was also a clear effect of expertise. The choices made by magicians with a limited amount of experience (i.e., 1 to 4 years of experience practising) did not significantly differ from the choices of nonmagicians. By contrast, magicians with more than 5 years of experience practising magic excelled at not being misled by the sleight of hand techniques presented. Remarkably, while both participants with none or little experience and experienced magicians excelled at choosing correctly when observing the real transfer emulating the palming technique, only participants with none or little experience did so when observing a real transfer emulating the French drop movements. In this case, experienced magicians appeared to have difficulties at correctly identifying the hand containing the coin.

While, as a group, magicians in Experiment 1 performed significantly better than non-magicians at identifying a magic effect, further inspections of the magician's experience revealed that only magicians that had trained in sleight of hand for more than 5 years were able to significantly choose the correct hand when observing a palm transfer technique. This five-year threshold is worth further exploration: one might wonder whether a magician must have mastered the sleight to be able to recognise it in others, or whether it is a case of having had more experience observing other magicians performing it.

These results are in accordance with most evidence substantiating the level of ability that deliberate practice across time in a particular domain can elicit (Farrington-Darby \& Wilson, 2006). However, it should be noted that these skills are not necessarily prompted by the number of years practising per se. Indeed, the opposite might be true in some capacity, the lower performance of individuals with years of experience auditing or performing medical diagnostics when compared to less experienced individuals shows that experience might not necessarily be related to expertise (Ericsson, 2008). As such, the expertise skill might be moderated by the type and quality of the practice, rather than the number of years (Ward et al., 2007). For example, in singers, amateurs tend to focus on emotional expression amongst other factors when practising, while expert professional singers tend to focus on precision and technique (Grape et al., 2002). Such differences in the type of practice might be the cause of the effects we see with magicians when identifying false movements. For example, expert magicians might be focusing more on making their movements as similar to real transfers as possible when practising, and this focus might be granting them the skills necessary to perceive the same technique when performed by others. The positive effect that spending extensive time deliberately practising a skill has on the level of expertise of the individual has been discussed in detail (for a review see Ericsson and Lehmann, 1996). Moreover, even for those with already significant experience, evidence suggests that on average, ten years practising a particular domain is necessary for both full expert dominion over it, and success at a professional level (Ericsson et al., 1993; Simon \& Chase, 1988; Bryan \& Harter, 1899). As such, further experimental evidence should investigate whether the level and quality of the practice that magicians undergo elicit a similar relationship in sleight of hand recognition than the one presented here.

When observing the real transfer of Experiment 1 all participants excelled at identifying the location of the coin regardless of their level of experience practising magic. This was expected as the movement itself reveals the location of the coin. When the experimenter performed the real transfer, the coin was tossed from one hand to the other, thus the observer physically sees the coin move. Moreover, as the movement 
is a common method of transferring an object from one hand to the other, which is mastered early in child development (Eppler, 1995; Hinojosa et al., 2003; Soska et al., 2010), it is reasonable to assume that any spectator with an unimpaired visual and attentional system would easily locate the hand in which the coin has been transferred to.

In Experiment 2 (French drop) similar patterns were found to those reported for Experiment 1 in terms of the magic effect condition, namely magicians did, as a group, perform significantly better than nonmagicians at identifying the sleight of hand. Non-magicians and magicians with less than 5 years of experience were significantly misled by the French drop technique, while more experienced magicians were able to recognise that the motion presented was mimed, and thus not a real transfer of object.

Regarding the real transfer counterpart in Experiment 2, non-magicians and magicians with little experience excelled at significantly choosing the correct hand, whilst there was no significant difference in the choices of experienced magicians. These results stand in contrast to the pattern found in Experiment 1, in which all participants (including experienced magicians) significantly chose the correct hand. To further explain these results, it is important to reiterate that there is a clear difference between the techniques used in Experiment 1 and in Experiment 2, not only methodologically but also regarding the amount of perceptual information that the techniques provide to the observer. In the palming technique used in Experiment 1, whilst there is a simulation of a normal transfer of objects, the movement is not ambiguous (i.e., with attentive observation, the observer can identify that the hand mimicking the transfer is not actually transferring the coin). Similarly, the real transfer technique used in Experiment 1 ofers visual clues to the attentive observer indicating that a real transfer has occurred (such as the visible drop of the coin from one hand to the other). This is not the case for either condition in Experiment 2, in which the index and middle finger fully cover the coin (Figure 1). As the coin is fully covered for both the initial grab of the coin and the retention of it, the observer cannot be certain whether the transfer of objects is real or not (as the coin cannot be seen either way).

The performance exhibited by experienced magicians in the real transfer counterpart of Experiment 2 may be explained by the magician's mindset rather than their discriminatory ability. Given the ambiguity of the movements observed, it is possible that magicians with several years practising how to fool others by utilising sleight of hand have a more pessimistic mindset than non and less experienced magicians when a motion does not provide clear and observable information. When observing an ambiguous movement, experienced magicians might consider the possibility of foul play and choose accordingly, whilst non-magicians and less experience magicians might not suffer from similar pessimistic perceptive biases. The superior ability of magicians to detect mimed movements from real ones has already been documented by Quarona et al., (2020), where magicians performed significantly better when discriminating the mimed motion of an individual reaching to grasp a glass than non-magicians. However, given our results, this superior ability seems to be developed after several years of training in the craft. Moreover, as evidenced by the experienced magicians' poor performance in the real French drop transfer, this prowess does not seem to grant the experienced magicians with a superior ability to 
discriminate between mimed and non-mimed movements when such movements are perceptually ambiguous.

It is important to note that, in this study, we took a broad-brush approach to magicians' expertise, and as such the 5-year threshold presented ought to be appraised with caution. Given that the experience level of the participants in this study was measured as four broad groups, the data does not offer insight into the experience variation within the groups (i.e., the exact number of years practising magic), or other variables that might have contributed to such differences in responses (such as the amount of time spent per day practising for example). Consequently, further research is merited to provide insight into the relationship between the number of years of deliberate practice of magicians, and their ability to discriminate deceptive movements.

Nonetheless, our results suggest that whilst, as a group, magicians are better at perceiving deceptive motions in contrast to non-magicians, this ability appears to be moderated by the quantity of experience practising magic: with expert magicians excelling at the recognition of deceptive movements, and nonmagicians and magicians with little experience being unable to recognise the fake transfers as deceptive actions. Interestingly the ability to distinguish mimed from real movements does not seem to grant the expert magicians any advantage when these motions are ambiguous in nature. In this case, nonmagicians and less experienced magicians seem to be more likely to be correct. We suggest that this might be because expert magicians who have heavily trained in the art of deception, and routinely use their movements to mislead a naïve audience, tend to adopt a pessimistic approach when a movement does not offer clear perceptive clues and consequently, they are more prone to suspect foul play even if the movements are genuine.

\section{Declarations}

\section{Ethical approval}

As the experiment did not involve the subjects disclosing any information about their individual identity, the participants were not from any vulnerable group, and the conditions of the experiment did not pose any risk to the participant, the experiment did not require ethical approval by the University of Cambridge. All procedures were performed in accordance with relevant guidelines.

\section{References}

1. Anders Ericsson, K. Deliberate practice and acquisition of expert performance: a general overview. Acad. Emerg. Med, 15 (11), 988-994 (2008).

2. Barnhart, A. S. The Exploitation of Gestalt Principles by Magicians. Perception, 39 (9), 1286-1289 https://doi.org/10.1068/p6766 (2010).

3. Calvo-Merino, B., Glaser, D. E., Grèzes, J., Passingham, R. E. \& Haggard, P. Action observation and acquired motor skills: An fMRI study with expert dancers. Cereb. Cortex, 
https://doi.org/10.1093/cercor/bhi007 (2005).

4. Camponogara, I., Rodger, M., Craig, C. \& Cesari, P. Expert players accurately detect an opponent's movement intentions through sound alone. Journal of Experimental Psychology: Human Perception and Performance, 43 (2), 348-359 https://doi.org/10.1037/xhp0000316 (2017).

5. Cavina-Pratesi, C., Kuhn, G., letswaart, M. \& Milner, A. D. The magic grasp: motor expertise in deception. PloS One, 6 (2), (2011). e16568

6. Charness, N., Tuffiash, M., Krampe, R., Reingold, E. \& Vasyukova, E. The role of deliberate practice in chess expertise. Applied Cognitive Psychology, 19 (2), 151-165 (2005).

7. Clayton, N. \& Wilkins, C. (2019). Magic and mysticism reveal cognitive shortcuts with implications beyond entertainment. https://www.repository.cam.ac.uk/handle/1810/293642

8. Cocchini, G., Galligan, T., Mora, L. \& Kuhn, G. The magic hand: Plasticity of mental hand representation. Quarterly Journal of Experimental Psychology, 71 (11), 2314-2324 https://doi.org/10.1177/1747021817741606 (2018).

9. Cui, J., Otero-Millan, J., Macknik, S., King, M. \& Martinez-Conde, S. Stronger perception of magic without social misdirection. Journal of Vision, 11 (11), 475 https://doi.org/10.1167/11.11.475 (2011).

10. Ekroll, V., Sayim, B. \& Wagemans, J. Against better knowledge: The magical force of amodal volume completion. I-Perception, 4 (8), 511-515 (2013).

11. Ekroll, V. \& Wagemans, J. Conjuring deceptions: Fooling the eye or fooling the mind? Trends in Cognitive Sciences, 20 (7), 486-489 (2016).

12. Eppler, M. A. Development of manipulatory skills and the deployment of attention. Infant Behavior and Development, 18 (4), 391-405 (1995).

13. Ericsson, K. \& Anders (2012). The Influence of Experience and Deliberate Practice on the Development of Superior Expert Performance. The Cambridge Handbook of Expertise and Expert Performance, 683-704. https://doi.org/10.1017/cbo9780511816796.038

14. Ericsson, K. \& Anders Deliberate practice and the acquisition and maintenance of expert performance in medicine and related domains. Acad. Med, 79 (10), S70-S81 (2004).

15. Ericsson, K., Anders, Krampe, R. T. \& Tesch-Römer, C. The role of deliberate practice in the acquisition of expert performance. Psychol. Rev, 100 (3), 363 (1993).

16. Ericsson, K., Anders \& Lehmann, A. C. Expert and exceptional performance: Evidence of maximal adaptation to task constraints. Annual Review of Psychology, 47 (1), 273-305 (1996).

17. Ericsson, K., Anders \& Smith, J. (1991). Prospects and limits of the empirical study of. Toward a General Theory of Expertise: Prospects and Limits, 1.

18. Ericsson, K. A. (1996). The acquisition of expert performance: an introduction to some of the issues.

19. Farrington-Darby, T. \& Wilson, J. R. The nature of expertise: A review. Appl. Ergon, 37 (1), 17-32 (2006). 
20. Gallese, V. \& Goldman, A. Mirror neurons and the mind-reading. Trens in Cognitive Sciences, https://doi.org/10.1016/S1364-6613(98)01262-5 (1998).

21. Galton, F. Hereditary genius (Macmillan and Company, 1869).

22. Garcia-Pelegrin, E., Schnell, A. K., Wilkins, C. \& Clayton, N. S. An unexpected audience., 369 (6510), 1424 LP - 1426https://doi.org/10.1126/science.abc6805 (2020).

23. Garcia-Pelegrin, E., Schnell, A. K., Wilkins, C. \& Clayton, N. S. (2021). Exploring the perceptual inabilities of Eurasian jays (Garrulus glandarius) using magic effects. Proceedings of the National Academy of Sciences, 118(24). https://doi.org/10.1073/PNAS.2026106118

24. Gaser, C. \& Schlaug, G. Brain structures differ between musicians and non-musicians. Journal of Neuroscience, 23 (27), 9240-9245 (2003).

25. Glaser, R. \& Chi, M. T. H. (1988). Overview, The Nature of Expertise, ed. MTH Chi, R. Glaser \& MJ Farr.LEA Hillsdale NJ.

26. Gobet, F. \& Charness, N. (2018). Expertise in chess.

27. Grape, C., Sandgren, M., Hansson, L. O., Ericson, M. \& Theorell, T. Does singing promote well-being?: An empirical study of professional and amateur singers during a singing lesson. Integrative Physiological \& Behavioral Science, 38 (1), 65-74 (2002).

28. Hegarty, M., Canham, M. S. \& Fabrikant, S. I. Thinking about the weather: How display salience and knowledge affect performance in a graphic inference task. Journal of Experimental Psychology: Learning, Memory, and Cognition, 36 (1), 37 (2010).

29. Hegarty, M., Keehner, M., Khooshabeh, P. \& Montello, D. R. How spatial abilities enhance, and are enhanced by, dental education. Learning and Individual Differences, 19 (1), 61-70 (2009).

30. Helm, F., Reiser, M. \& Munzert, J. (2016). Domain-Specific and Unspecific Reaction Times in Experienced Team Handball Goalkeepers and Novices. In Frontiers in Psychology (Vol. 7, p. 882). https://www.frontiersin.org/article/10.3389/fpsyg.2016.00882

31. Hinojosa, T., Sheu, C. \& Michel, G. F. Infant hand-use preferences for grasping objects contributes to the development of a hand-use preference for manipulating objects. Developmental Psychobiology: The Journal of the International Society for Developmental Psychobiology, 43 (4), 328-334 (2003).

32. Kanizsa, G. \& Gerbino, W. (1982). Amodal completion: Seeing or thinking?

33. Kirkman, M. A. Deliberate practice, domain-specific expertise, and implications for surgical education in current climes. Journal of Surgical Education, 70 (3), 309-317 (2013).

34. Kuhn, G., Amlani, A. A. \& Rensink, R. A. Towards a science of magic. Trends in Cognitive Sciences, 12 (9), 349-354 (2008).

35. Kuhn, G., Caffaratti, H. A., Teszka, R. \& Rensink, R. A. A psychologically-based taxonomy of misdirection. Frontiers in Psychology, 5 (DEC), 1-14 https://doi.org/10.3389/fpsyg.2014.01392 (2014).

36. Kuhn, G. \& Land, M. F. There's more to magic than meets the eye. Curr. Biol, 16 (22), 950-951 https://doi.org/10.1016/j.cub.2006.10.012 (2006). 
37. Mann, D. Y., Williams, A. M., Ward, P. \& Janelle, C. M. Perceptual-cognitive expertise in sport: A metaanalysis. Journal of Sport and Exercise Psychology, 29 (4), 457-478 https://doi.org/10.1123/jsep.29.4.457 (2007).

38. Michotte, A., Thinès, G. \& Crabbé, G. (1991). Amodal completion of perceptual structures.Michotte's Experimental Phenomenology of Perception,140-167.

39. Moore, L. J., Harris, D. J., Sharpe, B. T., Vine, S. J. \& Wilson, M. R. Perceptual-cognitive expertise when refereeing the scrum in rugby union. Journal of Sports Sciences, 37 (15), 1778-1786 https://doi.org/10.1080/02640414.2019.1594568 (2019).

40. Morreale, F., Masu, R., De Angeli, A. \& Fava, P. (2013). The effect of expertise in evaluating emotions in music. The 3rd International Conference on Music \& Emotion, Jyväskylä, Finland, June 11-15, 2013.

41. Otero-Millan, J., Macknik, S. L., Robbins, A. \& Martinez-Conde, S. Stronger misdirection in curved than in straight motion. Frontiers in Human Neuroscience, 5, 133 (2011).

42. Quarona, D. et al. A kind of magic: Enhanced detection of pantomimed grasps in professional magicians. Quarterly Journal of Experimental Psychology, 73 (7), 1092-1100 (2020).

43. Rizzolatti, G., Fogassi, L. \& Gallese, V. Neurophysiological mechanisms underlying the understanding and imitation of action. Nature Reviews Neuroscience, https://doi.org/10.1038/35090060 (2001).

44. Schall, R. Estimation in generalized linear models with random effects., 78 (4), 719-727 (1991).

45. Shipley, T., Manduca, C., Resnick, I. \& Schilling, C. (2009). Expertise in spatial visualization: Can geologists reverse time. Psychonomic Society.

46. Simon, H. \& Chase, W. (1988). Skill in chess. In Computer chess compendium (pp. 175188).Springer.

47. Soska, K. C., Adolph, K. E. \& Johnson, S. P. Systems in development: motor skill acquisition facilitates three-dimensional object completion. Dev. Psychol, 46 (1), 129 (2010).

48. Stull, A. T., Hegarty, M., Dixon, B. \& Stieff, M. Representational translation with concrete models in organic chemistry. Cognition and Instruction, 30 (4), 404-434 (2012).

49. Thomas, C. \& Didierjean, A. No need for a social cue! A masked magician can also trick the audience in the vanishing ball illusion. Atten. Percept. Psychophys, 78 (1), 21-29 (2016).

50. Ward, P., Hodges, N. J., Starkes, J. L. \& Williams, M. A. The road to excellence: Deliberate practice and the development of expertise. High Ability Studies, 18 (2), 119-153 (2007).

51. William, L. B. \& Harter, N. Studies on the telegraphic language: The acquisition of a hierarchy of habits. Psychol. Rev, 6 (4), 345 (1899).

52. Williams, A. M., Ward, P., Knowles, J. M. \& Smeeton, N. J. Anticipation skill in a real-world task: Measurement, training, and transfer in tennis. Journal of Experimental Psychology: Applied, 8 (4), 259-270 https://doi.org/10.1037/1076-898X.8.4.259 (2002).

53. Wöllner, C. \& Cañal-Bruland, R. Keeping an eye on the violinist: Motor experts show superior timing consistency in a visual perception task. Psychol. Res, 74 (6), 579-585 
https://doi.org/10.1007/s00426-010-0280-9 (2010).

\section{Figures}

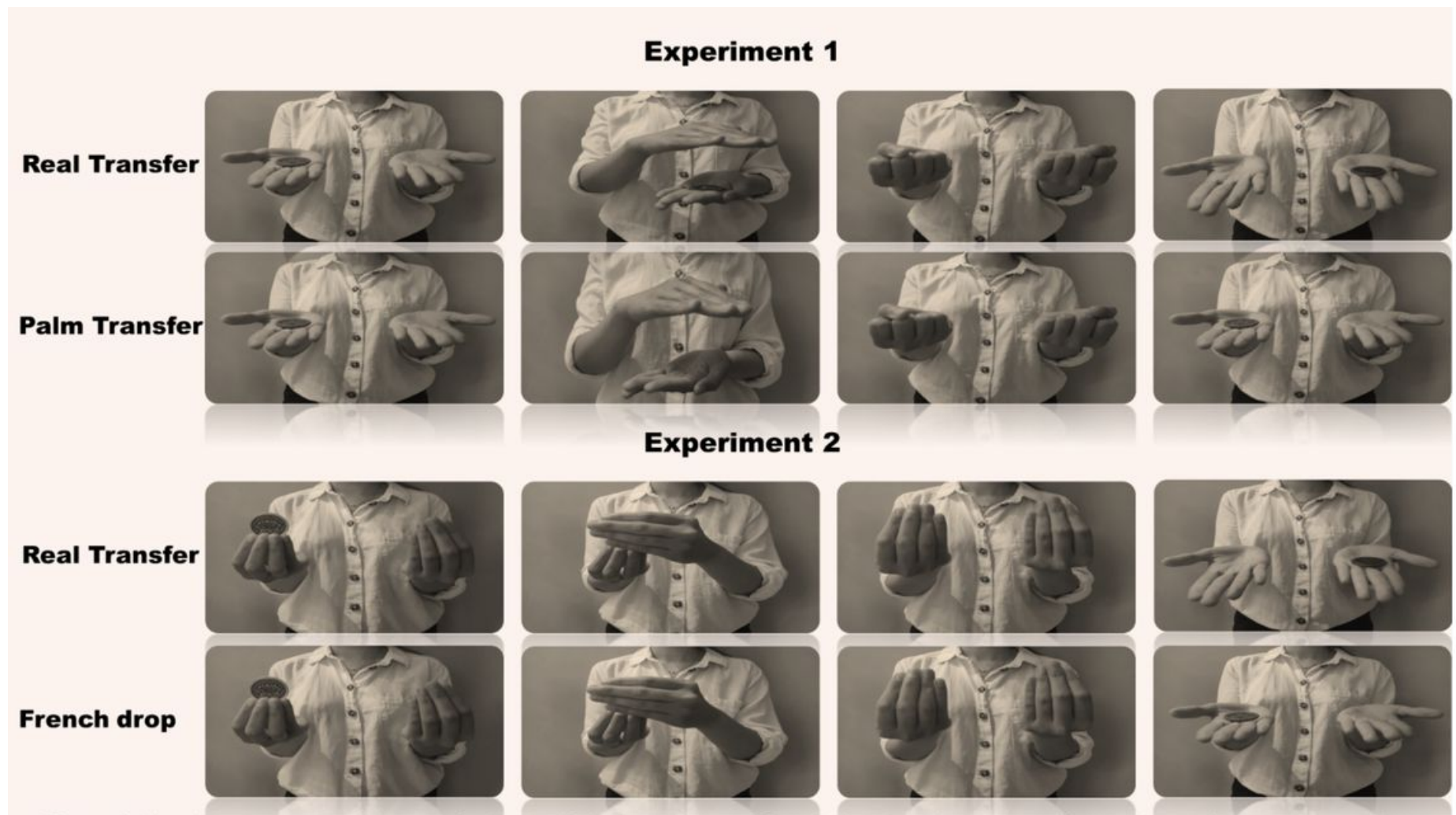

Figure 1. Hand movements of the magic effects (Palm transfer and French drop) and corresponding real transfer counterparts for Experiment 1 and 2.

\section{Figure 1}

Hand movements of the magic effects (Palm transfer and French drop) and corresponding real transfer counterparts for Experiment 1 and 2. 


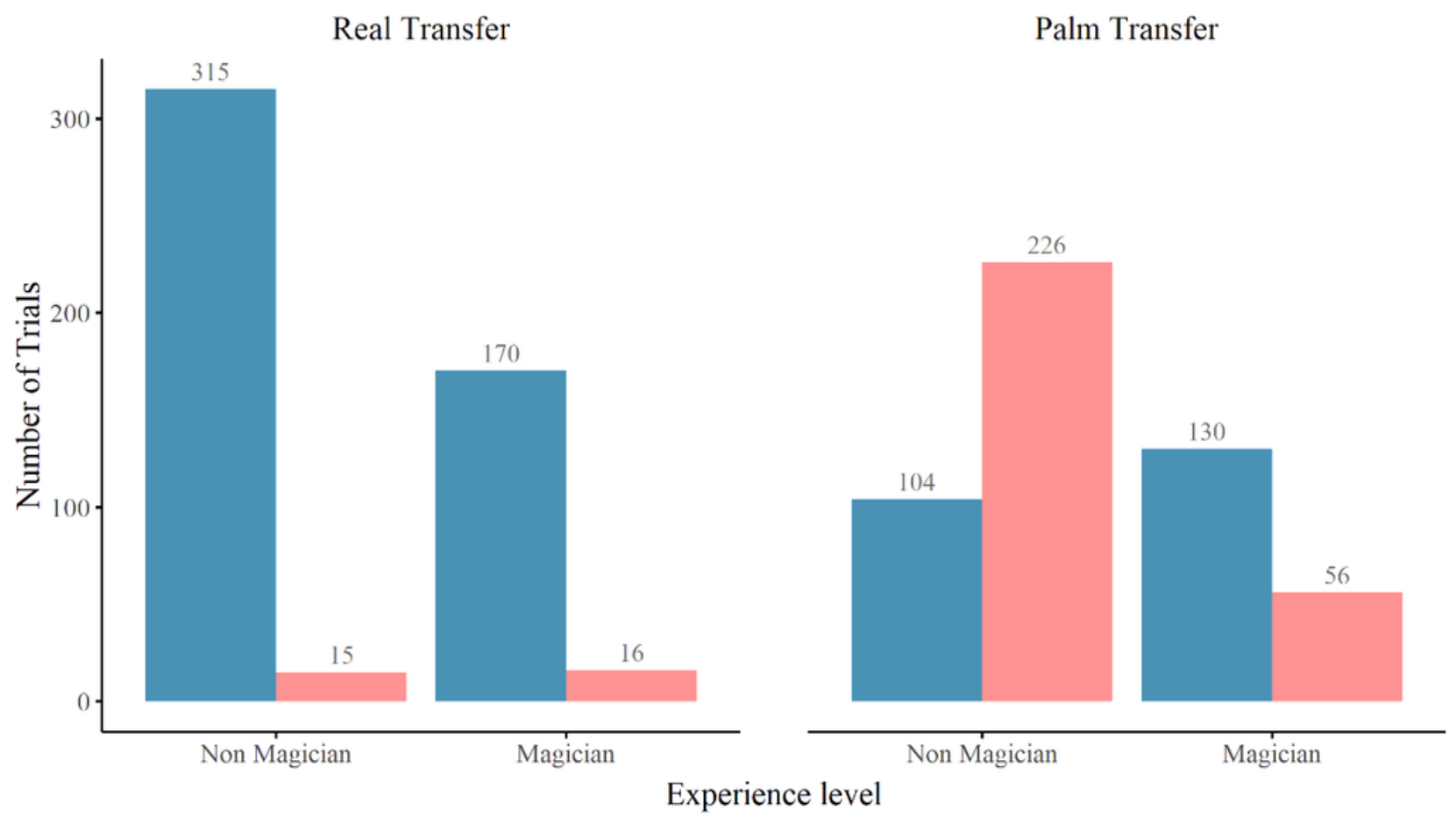

Hand Choices

Correct

Incorrect

Figure 2

Choices of non-magicians and magicians in palm transfer and real transfer counterpart

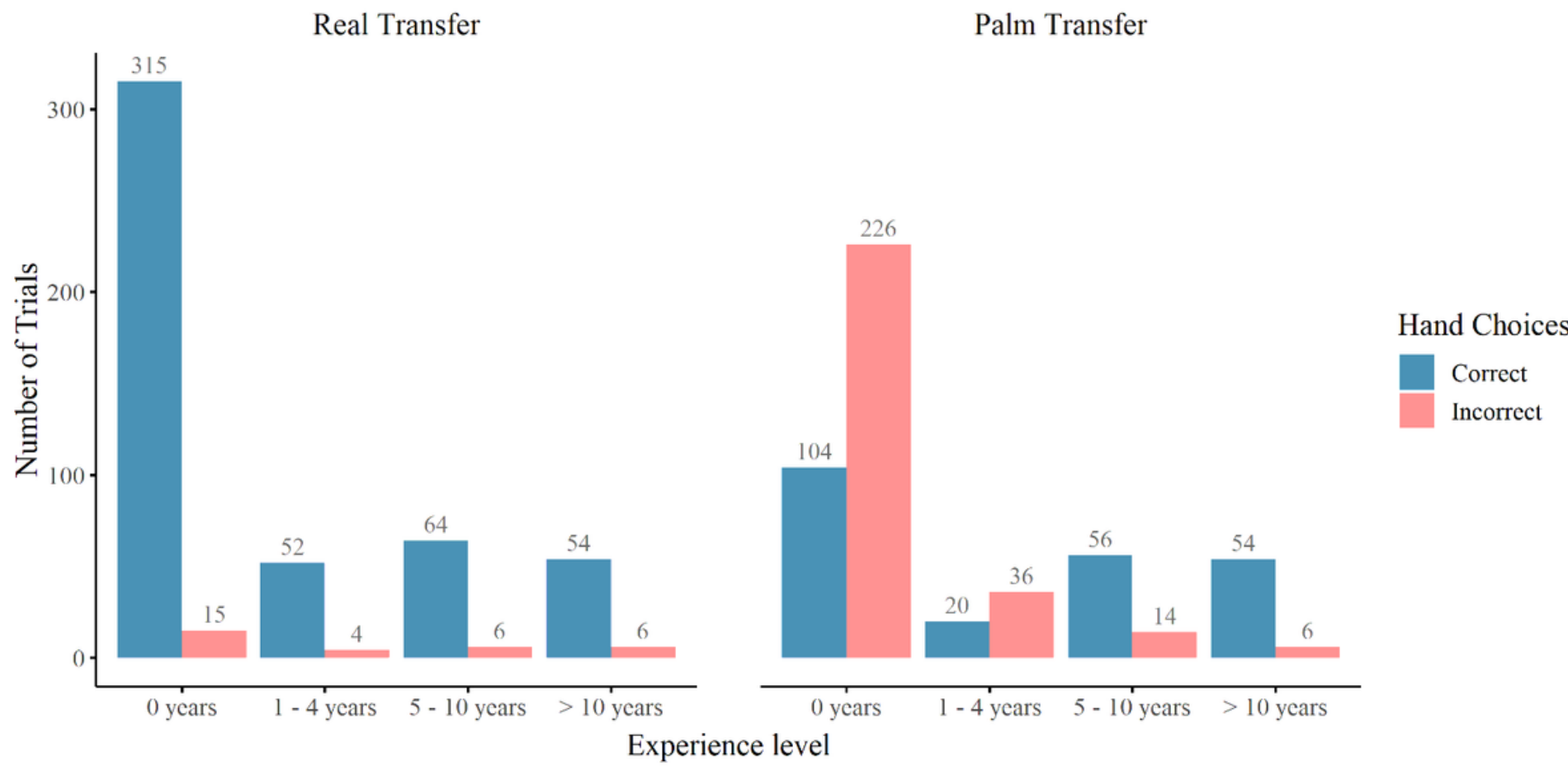

\section{Figure 3}

Choices in palm transfer, and real transfer counterpart separated by levels of experience practicing magic. 


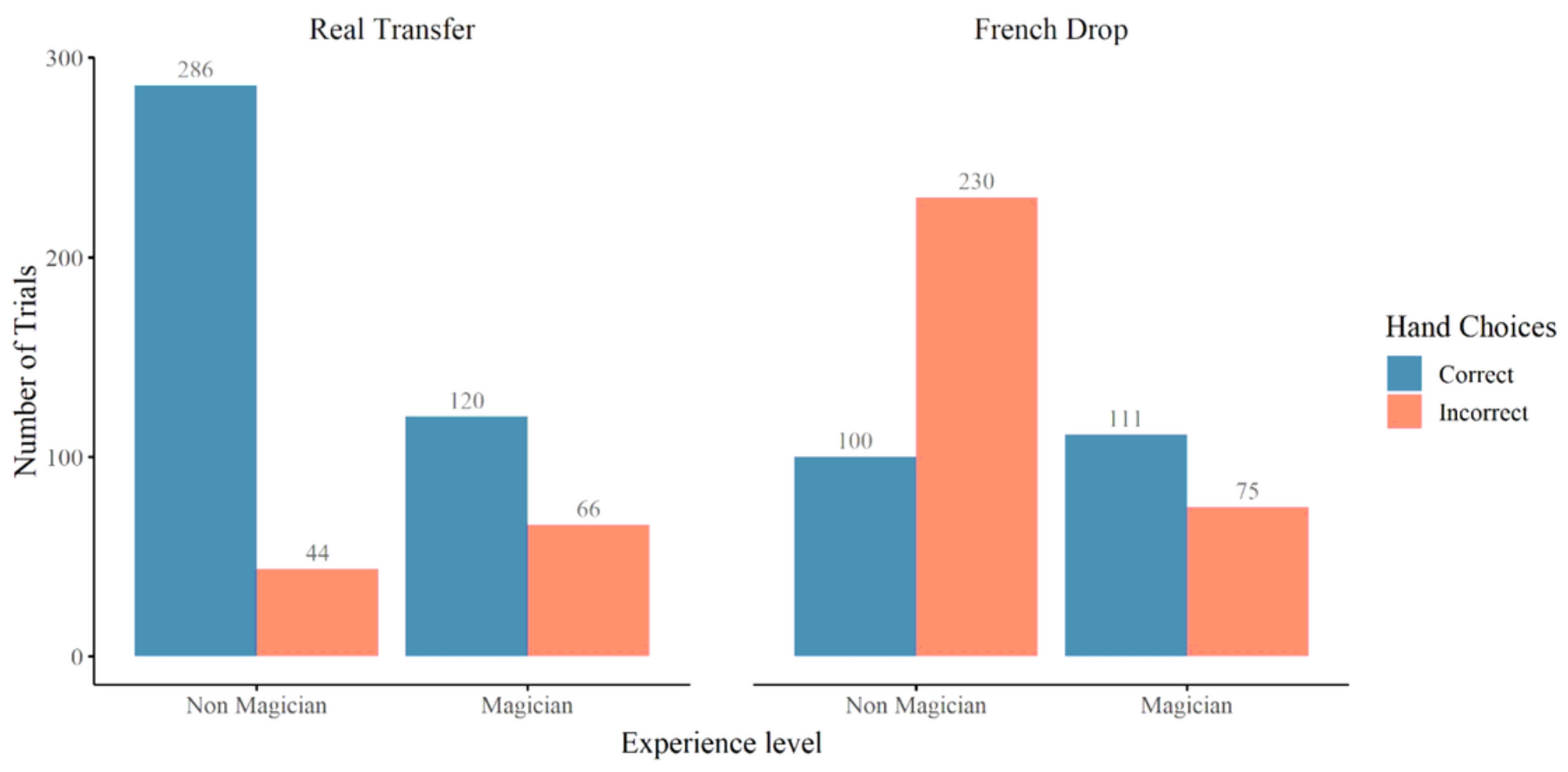

Figure 4. Choices of Non-magicians and magicians in French drop and real transfer counterpart.

\section{Figure 4}

Choices of Non-magicians and magicians in French drop and real transfer counterpart.

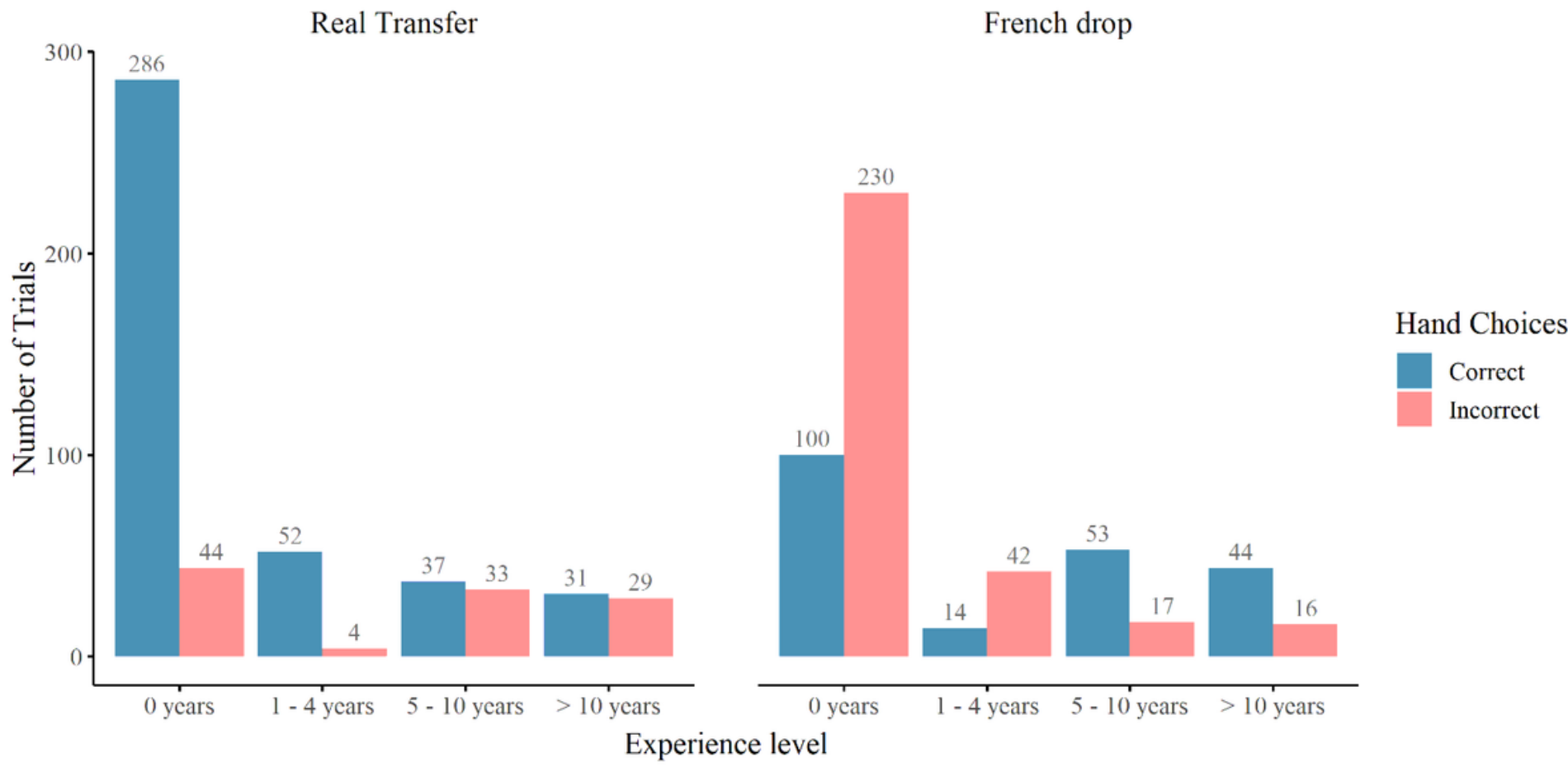

\section{Figure 5}

Choices in French drop, and real transfer counterpart separated by levels of experience practicing magic. 


\section{Supplementary Files}

This is a list of supplementary files associated with this preprint. Click to download.

- SuplementarymaterialVideoconditions.mp4 